This material is published in the open archive of Mid Sweden University DIVA http://miun.divaportal.org to ensure timely dissemination of scholarly and technical work.

Copyright and all rights therein are retained by authors or by other copyright holders. All persons copying this information are expected to adhere to the terms and constraints invoked by each author's copyright. In most cases, these works may not be reposted without the explicit permission of the copyright holder.

Damghanian, M.; Olsson, R.; Sjöström, M., "Extraction of the lateral resolution in a plenoptic camera using the SPC model," 3D Imaging (IC3D), 2012 International Conference on, vol., no., pp.1,5, 3-5 Dec. 2012.

DOI: 10.1109/IC3D.2012.6615137

(C) 2013 IEEE. Personal use of this material is permitted. However, permission to reprint/republish this material for advertising or promotional purposes or for creating new collective works for resale or redistribution to servers or lists, or to reuse any copyrighted component of this work in other works must be obtained from the IEEE. 


\title{
EXTRACTION OF THE LATERAL RESOLUTION IN A PLENOPTIC CAMERA USING THE SPC MODEL
}

\author{
Mitra Damghanian, Roger Olsson, Mårten Sjöström \\ Department of Information Technology and Media \\ Mid Sweden University \\ Sundsvall
}

\begin{abstract}
Established capturing properties like image resolution need to be described thoroughly in complex multidimensional capturing setups such as plenoptic cameras (PC), as these introduce a trade-off between resolution and features such as field of view, depth of field, and signal to noise ratio. Models, methods and metrics that assist exploring and formulating this trade-off are highly beneficial for study as well as design of complex capturing systems. This work presents how the important high-level property lateral resolution is extracted from our previously proposed Sampling Pattern Cube (SPC) model. The SPC carries ray information as well as focal properties of the capturing system it models. The proposed operator extracts the lateral resolution from the SPC model throughout an arbitrary number of depth planes resulting in a depth-resolution profile. We have validated the resolution operator by comparing the achieved lateral resolution with previous results from more simple models and from wave optics based Monte Carlo simulations. The lateral resolution predicted by the SPC model agrees with the results from wave optics based numerical simulations and strengthens the conclusion that the SPC fills the gap between ray-based models and wave optics based models, by including the focal information of the system as a model parameter. The SPC is proven a simple yet efficient model for extracting the depthbased lateral resolution as a high-level property of complex plenoptic capturing system.
\end{abstract}

Index Terms - Camera modeling, plenoptic camera, lateral resolution, sampling pattern cube.

\section{INTRODUCTION}

Established capturing properties like image resolution need to be described thoroughly in complex multidimensional capturing setups such as plenoptic cameras. This investigation is required both to understand trade-offs among various quantities such as spatial, angular or depth resolution between unconventional capturing systems as well as exploring each system behavior individually. Light field sampling behavior of an optical system, and hence its associated features such as the captured resolution, can vary for different depth planes or different distances from the main optical axis. Adjustments in the system, or unintended variations in the capturing system properties, are other sources for variations in sampling behavior and therefore in the high-level properties of the system. It is beneficial to have a model that provides straightforward extraction of features with a desired level of details, when analyzing, designing and using complex capturing systems. This knowledge can also be used for developing, rendering and post processing approaches or adjusting prior computational methods for new device setups. Resolution in plenoptic cameras is an example that asks for more detailed investigations, which considers the properties of the capturing system. Such investigations of complex capturing system has been the subject of prior and recent works $[1,2,3]$.

Models have been proposed that describe the light field and how it is sampled by different image capturing systems $[4,5]$. The light field model which is a simplified representation of the plenoptic function (with one less dimension) has proven useful for applications spanning computer graphics, digital photography, and 3D reconstruction. The scope of the light field has also been broaden by employing wave optics to model diffraction and interference [6] where the resulting augmented light field gain more explainability at the expense of increased model complexity. Our previously proposed sampling pattern cube (SPC) model is utilized in this work and contrary to the previously proposed ray-based models, it includes focus information at a more simple way than the wave optics model [7]. Focus information is a vital feature for inferring high-level properties such as lateral resolution in different depth planes. Being able to easily quantify such properties is of practical use for conventional image capturing systems in general but of specific interest when working with more complex systems such as camera arrays [8] and light field or plenoptic cameras [9].

In this work we have proposed how to extract lateral resolution from the SPC model, validated the resolution extraction operator with respect to wave optics based Monte Carlo simulations, and related the results with spatial resolution, which is a high-level property of main interest. In the following parts, 
we first give a general overview of the SPC model in Section 2 and then we define the lateral resolution operator in this model. Section 3 introduces the plenoptic camera system that we model as an SPC and apply the resolution operator to. Section 4 illustrates the obtained results from the SPC model followed by a discussion and comparison with results from other models. Finally, Section 5 concludes the work with a hint on possible future interests.

\section{THE SPC MODEL}

The SPC is a geometry-based model for the optical capturing systems. Light samples in this model are in the form of light containers (LCs) defined by a tip position $\left(x_{C}, y_{C}, z_{C}\right)$ and an angular span $\left(\phi_{s}, \phi_{f}, \theta_{s}, \theta_{f}\right)$ :

$$
L C:=\left\{\begin{array}{l}
\left(x_{C}, y_{C}, z_{C}\right) \\
\left(\phi_{s}, \phi_{f}, \theta_{s}, \theta_{f}\right)
\end{array}\right.
$$

and

$$
S P C:=\left\{L C_{i}\right\}, i=1, \ldots, k .
$$

Figure 1 shows an LC in 3D space and we stick to a rectangular base shape for simple illustration purposes. LCs represent the form of in-focus light and so the SPC model preserves the focal properties of the capturing system in a compact but at the same time comprehensive way. To generate the SPC model of a capturing system, we start from the initial set of LCs with their tip position at the center of each pixel on the sensor plane and an angular span equal to the light acceptance angle of each sensor pixel. Then we backtrack each LC through the optical system passing elements such as apertures and lenses, which will transform the initial set of LCs into new sets using geometrical optics. The transformations continue until we reach a final set of LCs with new tip positions and angular span that carry the sampling properties of the capturing system. This final set of LCs and their correspondence to the initial sensor pixels build the SPC model of the system and preserve the focal information and the information about where each recorded light sample on the sensor cell is originating from.

The base $B$ of an LC on plane $z=z_{0}$ is defined as its projection on the plane (or the intersection area of that LC with the depth plane $\left.z=z_{0}\right)$ :

$$
B\left[L C, z_{0}\right]:=\left(x_{1}, x_{2}, y_{1}, y_{2}, z_{0}, \phi_{s}, \phi_{f}, \theta_{s}, \theta_{f}\right)
$$

where

$$
\begin{aligned}
& x_{1,2}=x_{C}+\left(z_{C}-z_{0}\right) \tan \phi_{s, f} \\
& y_{1,2}=y_{C}+\left(z_{C}-z_{0}\right) \tan \theta_{s, f}
\end{aligned}
$$

\subsection{Feature extraction}

Some of the high-level properties of the optical capturing system and how they are reflected in the SPC model are investigated in [7]. The focal plane of the system is one feature

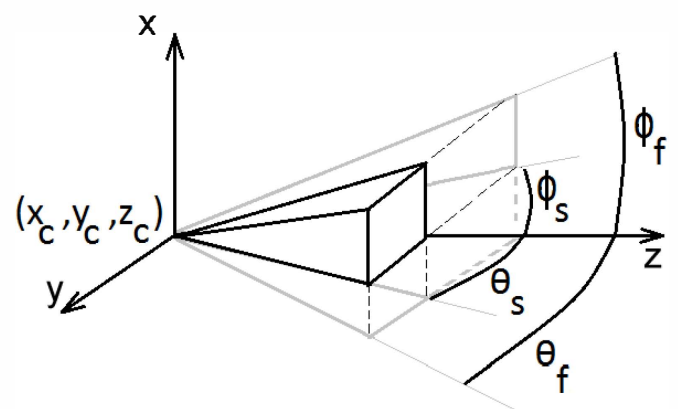

Fig. 1. Illustration of an LC in 3D space

easily obtained from the information in the SPC model: It is the depth value $(\mathrm{z})$ of the tip position of the final set of LCs. Other features such as spatial and angular resolution, as well as how variations in the pitch of the lenslet or focal length of the main lens in a plenoptic camera affect the SPC model, are also qualitatively discussed in [7]. In the work presented here, we introduce a quantitative operator to derive the lateral resolution in different depth planes. The aim is to investigate the capability of the SPC model in extracting high-level properties of a capturing system. We later apply the defined operator to an exemplary plenoptic capturing system and evaluate the operator by comparing the results to those from ray based model and wave optics based Monte Carlo simulations for the same capturing system.

\subsection{Lateral resolution in the SPC model}

Lateral resolution for a complex plenoptic capturing system is generally defined as the inverse of the minimum distance (min-dist) between two resolvable points located at a specific depth plane within the system's common field of view (CFV). Depending on the purpose of lateral resolution analysis, the number and locations of depth planes may be arbitrarily chosen.

To extract lateral resolution using the SPC model we need to apply proper adjustments to the general definition and incorporate the specific features of the model. Two approaches are implemented here based on two different definitions of the lateral resolution in a SPC model. Definition I: This simpler operator defines the inverse of the lateral resolution value as the maximum Euclidian distance between immediate neighboring LCs' base center points. Figure 2 shows an example of neighboring LCs and their respective base area and center point. Two LCs are immediate neighbors if no other LC's base center point is located between their base center points. In the Definition I no focal property is used and the 


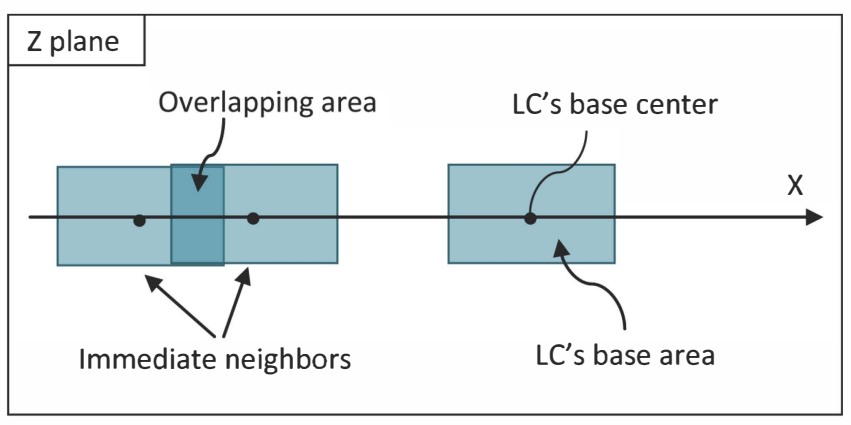

Fig. 2. Illustration of the LC's base area and center point

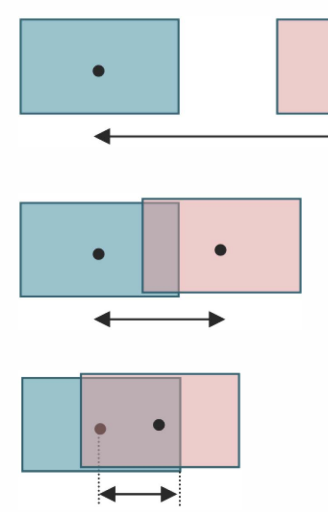

Case 1

min_dist $=$ center_dist

Fig. 3. Finding min-dist value

lateral resolution operator for all practical purposes reduce the SPC model to a ray model as only the principal rays passing through the LCs' centers are used to investigate the capturing system properties.

Definition II: The second and more elaborate operator utilizes the SPC model's focal information, which is preserved as the width of the LCs at each depth plane. This definition considers the freedom of movement of a point inside an LC's base area. The inverse of the lateral resolution value at a certain depth plane is defined as the largest of the following values: 1) maximum distance between center points of two immediate neighbor LCs, or 2) half of the LC's base width at that depth plane.

The following assumptions are made to make the proposed measure reflect the actual resolution:

- The resolution test points can only be located where system can sample them, which means inside the LCs in the SPC model (A consequence of the sparse sampling model).

- Two points are resolvable only if they are located within two different LCs.
Table 1. Camera system specifications

\begin{tabular}{lc} 
Lenslet array size & $11 \times 11$ \\
Lenslet focal length, $f$ & $12 \mathrm{~mm}$ \\
Lenslet pitch & $4.2 \mathrm{~mm}$ \\
Lenslet f-number & 22 \\
Spacing between lenslet array and image sensor, $g$ & $12.507 \mathrm{~mm}$ \\
Image sensor pixel number behind one lenslet & $251 \times 251$ \\
\hline
\end{tabular}

- Two LCs are considered different if they are not including each other's centers.

- The distance between two LCs is defined as the distance between their center points (center-dist).

To find the min-dist valid for the whole CFV we look for the worst case, i.e. we consider the two immediate neighbor LCs that have the maximum distance from each other. Then either of the following cases holds:

Case 1 If these two immediate neighbor LCs are not overlapping, then min-dist is equal to center-dist.

Case 2 If these two immediate neighbor LCs are overlapping but they are still different LCs, then the min-dist is equal to the center-dist.

Case 3 If these two immediate neighbor LCs are overlapping and they include each other's centers then all the LCs in the SPC are overlapping and the min-dist is equal to the half of the LC's width.

The process to find min-dist value is presented in Figure 3 where the two illustrated base areas belong to the two immediate neighbor LCs with maximum distance between their center points.

We will compare the level of explainability of the two above definitions in Section 4, and also compare their results with those from wave optics based Monte Carlo simulations.

\section{TEST SETUP}

We have considered a plenoptic capturing system with specifications given in Figure 4 and Table 1 when applying the defined resolution operators. In addition, we consider pixels behind each lenslet to be optically decoupled from any neighboring lenslets.

We first generate the SPC model of the capturing system with details in Table 1, as described in [7]. The two versions of the resolution extraction operator (Definition I and II) are then applied to the achieved SPC model. Symmetry in the defined capturing system makes analysis of the resolution values identical in both $\mathrm{x}$ and $\mathrm{y}$ dimensions. Moreover, the resolution analysis only considers the space directly in front of the 


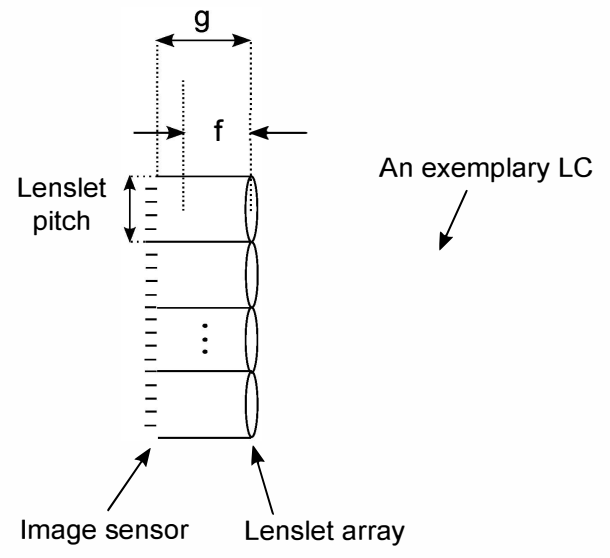

Fig. 4. Illustration of the test setup

lenslet system and discards the effect of the main lens, as that part of the capturing system merely acts as a relay to bring far objects closer to the lenslets by a magnification factor.

As the reference for our resolution operators we use wave optics based Monte Carlo simulations results produced by the 3D Imaging and Display Group at the University of Valencia [3]. This data whose validity is also confirmed by physical experiments is considered the ground-truth of the evaluated system in terms of lateral resolution.

\section{RESULTS AND DISCUSSION}

Figure 5 shows the minimum resolvable distances, or the inverse of the lateral resolution for a range of depth planes, obtained from the two operators. The data from wave optics based Monte Carlo simulations is also provided as reference data for comparison purposes. The focal plane of the system under the test is about $300 \mathrm{~mm}$ and the data at depth planes further away than this is presented. We apply a correction factor of two to the result from the two operators, relative to the wave optics based data. This takes into consideration the difference between the two methods were the former extracts resolvability using dark-light points and the later dark-lightdark points.

A general trend is observed in all the graphs which indicates there are depth planes where the lateral resolution drops (location of peaks in min-dist) compared to depth planes slightly closer or further. In these depth planes, LCs overlap and form clusters and the lateral resolution is decreased as a result of the poorer distribution of the LCs. It is observed that the resolution operator equal to ray-based model results, which is using only the distance of the LCs' centers, can set only an upper limit on the lateral resolution in different depth planes. We call this value the upper limit of the resolution since it is assuming the ideal case where all the information is carried by the center of the LC (or the single ray). Note that the SPC model allows for producing results equal to a ray-

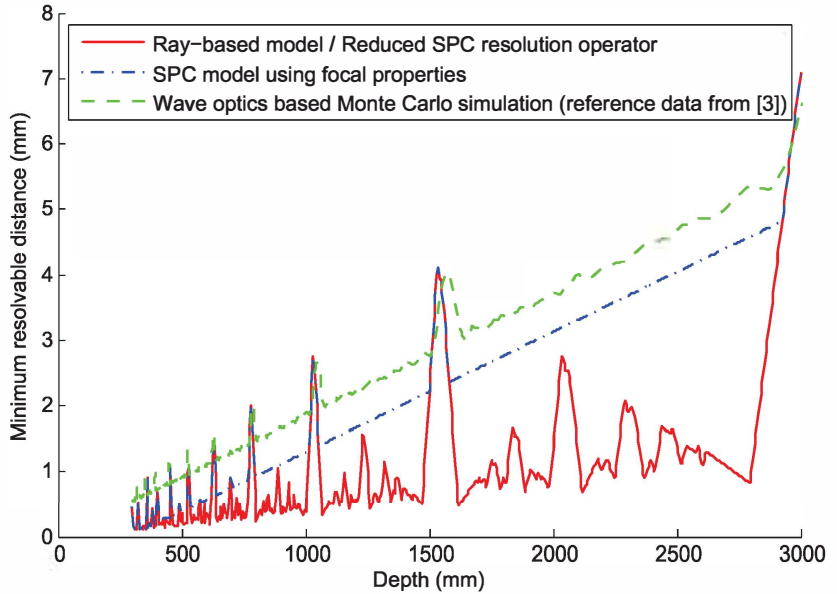

Fig. 5. The minimum resolvable lateral distance at different depth planes achieved from the ray-based model, the proposed SPC operator using focal properties, and the reference data from wave optics based Monte Carlo simulations.

based model, making the latter a subset of the SPC. Results from the Definition I operator are aligned with the wave optics based Monte Carlo simulations results in terms of the location of the peaks and periodicity of the depth planes with lowresolution, or min-dist peaks. However, the general slant of the results from Definition I operator and values at the intermediate depth planes are not matching with the results from the wave optics based Monte Carlo simulations.

We can see in Figure 5 that by using the proposed resolution operator (Definition II) which is based on the complete LC properties, the gap between upper limit of the resolution (from the Definition I operator) and its amount from the wave optics based Monte Carlo simulations is considerably reduced. Using LC properties, the resolution values from the proposed resolution operator are better in line with the Monte Carlo simulations data. Graphs agree in location of peaks, maximum amplitudes as well as the general slant at the intermediate depth planes. A gap still exists between the proposed operator results that consider the full SPC properties and the ground-truth reference data. This gap can be justified by different resolution thresholds considered in the SPC and Monte Carlo simulations. There are also simplifications assumed in the SPC model compared to the real system performance as the current SPC excludes noise, diffraction, and point spread function effects of the system. Detailed investigations of the above contributors to the lateral resolution extracted by the SPC model could be a subject of the future works.

\section{CONCLUSION}

We investigated lateral resolution as a high level property of the capturing system using the SPC model. We introduced an operator that leverage on all SPC features to extract the lat- 
eral resolution in each depth plane and applied it to the SPC model of a plenoptic camera setup. Comparing the achieved lateral resolution with previous results from the wave optics based Monte Carlo numerical approach, the SPC is proven a simple yet efficient model for extracting high-level properties of the capturing system. We conclude that the SPC model can predict the lateral resolution much closer to the actual resolution values compared to the ray-based model and this strengthens the SPC model as a tool capable of evaluating complex plenoptic capture systems. Next step is to introduce additional feature extractors in the SPC model for other properties of interest such as depth of field, angular and depth resolution, and validate and evaluate the introduced operators by applying them to complex capturing setups.

\section{ACKNOWLEDGMENT}

The authors would like to thank Héctor Navarro Fructuoso at the 3D Imaging and Display Group of University of Valencia for providing the wave optics based Monte Carlo simulations data used as a reference in this work's resolution comparison. This work has been supported by grant 2009/0264 of the KK Foundation, Sweden, by grant 00156702 of the EU European Regional Development Fund, Mellersta Norrland, Sweden, and by grant 00155148 of Länsstyrelsen i Västernorrland, Sweden.

\section{REFERENCES}

[1] H. Hoshino, F. Okano, H. Isono, and I. Yuyama. Analysis of resolution limitation of integral photography. J. Opt. Soc. Am. A, 15(8):2059-2065, Aug 1998.

[2] Zahra Kavehvash, Khashayar Mehrany, Saeed Bagheri, Genaro Saavedra, Hector Navarro, and Manuel MartinezCorral. 3d resolution in computationally reconstructed integral photography. volume 8384 , page 838417 . SPIE, 2012.

[3] Manuel Martinez-Corral, Hector Navarro, Emilio Sanchez-Ortiga, Genaro Saavedra, Anabel Llavador, Adrian Dorado, and Bahram Javidi. Non-homogeneity of lateral resolution in integral imaging. Journal of Display Technology, 2012.

[4] Steven J. Gortler, Radek Grzeszczuk, Richard Szeliski, and Michael F. Cohen. The lumigraph. In Proceedings of the 23rd annual conference on Computer graphics and interactive techniques, SIGGRAPH '96, pages 4354, New York, NY, USA, 1996. ACM.

[5] Marc Levoy and Pat Hanrahan. Light field rendering. In Proceedings of the 23rd annual conference on Computer graphics and interactive techniques, SIGGRAPH '96, pages 31-42, New York, NY, USA, 1996. ACM.
[6] Se Baek Oh, Sriram Kashyap, Rohit Garg, Sharat Chandran, and Ramesh Raskar. Rendering wave effects with augmented light field. Computer Graphics Forum, 29(2):507-516, 2010.

[7] Mitra Damghanian, Roger Olsson, and Mårten Sjöström. The sampling pattern cube - a representation and evaluation tool for optical capturing systems. In Advanced Concepts for Intelligent Vision Systems (ACIVS 2012), Brno, Czech Republic, Sep. 2012.

[8] Bennett Wilburn, Neel Joshi, Vaibhav Vaish, Eino-Ville Talvala, Emilio Antunez, Adam Barth, Andrew Adams, Mark Horowitz, and Marc Levoy. High performance imaging using large camera arrays. ACM Trans. Graph., 24(3):765-776, July 2005.

[9] Edward H. Adelson and John Y. A. Wang. Single lens stereo with a plenoptic camera. IEEE Transactions on Pattern Analysis and Machine Intelligence, 14:99-106, 1992. 